\title{
Serological test is an efficient supplement of RNA detection for
}

\section{confirmation of SARS-CoV-2 infection}

\author{
Ningshao Xia ${ }^{1}$, Guiqiang Wang ${ }^{2}$, Wenfeng Gong ${ }^{3}$ \\ ${ }^{1}$ School of Public Health, Xiamen University; ${ }^{2}$ Peking University First Hospital,; ${ }^{3}$ Bill \& Melinda \\ Gates Foundation \\ Corresponding author: Ningshao Xia, Email: nsxia@xmu.edu.cn; Guiqiang Wang, Email: \\ john131212@sina.com; Wenfeng Gong, Email: Wenfeng.Gong@gatesfoundation.org
}

\begin{abstract}
Until now, viral RNA detection is almost the only way to confirm the infection of SARS-CoV-2 in practice. But varied reasons lead to the low sensitivity by RNA detection, which proposes serious challenge to disease control. We tested the performance of detecting total antibody(Ab) and $\operatorname{IgM}$ antibody in serum by the methods of chemiluminescence, Enzyme Linked Immunosorbent Assay(ELISA), and colloidal gold. Data showed that the sensitivity and specificity for total Ab and IgM detection were high by all the three methods, and compared with IgM, the sensitivity was higher for total $\mathrm{Ab}$ detection. Evidence from studies showed viral RNA testing combining with serological testing could increase the sensitivity of diagnosis, while remaining the high specificity. Specific serolody test for SARS-CoV-2 has great value for clinical practice and public health.
\end{abstract}

Keywords : SARS-CoV-2; diagnosis; antibody; serology; screening

On March 7, 2020, The World Health Organization (WHO) declared that beyond 90 countries/territories/areas reported more than 100,000 cases (including 80,000 cases from China) and more than 3,400 deaths of COVID-19. ${ }^{[1]}$ Given the rapid spread of COVID-19, WHO raised the risk level of COVID-19 from "high" to "very high" on February 28, 2020, and call on all countries to give the highest priority to the prevention and control of COVID-19 epidemics. ${ }^{[2]}$ Currently, viral RNA detection by quantitative real-time polymerase chain reaction (PCR) and/or sequencing is almost the only way to confirm the diagnosis of SARS-CoV-2 infection in practice. However, the reported positive rate of PCR in COVID-19 patients was not high, and patients often need to be sampled several times and at multiple sites before final diagnosis. Some highly suspected patients who were epidemiologically linked to SARS-CoV-2 exposure strongly and with typical lung radiological findings remained RNA negative in their upper respiratory tract samples. Besides, RNA detection requires high-quality swab specimen. Consequently, these troubles propose serious challenge to disease control and preventive quarantine.

In addition, patients with mild symptom, asymptomatic infection, and those who are 
within the latent period of the disease cannot be observed clinically, which will become a major threat for COVID-19 prevention and control. Guan et al. analyzed the clinical characteristics of 1,099 patients with laboratory-confirmed COVID-19 from 552 hospitals in 30 provinces, autonomous regions, and municipalities in China, and found that radiographic or CT abnormality wasn't presented in 157 of 877 patients (17.9\%) with nonsevere disease, and only $87.7 \%$ and $67.8 \%$ of patients experienced typical symptoms such as fever and cough during hospitalization. ${ }^{[3]}$ Another study suggested that among 72,314 patients, 889 patients (1\%) diagnosed by positive viral nucleic acid test result were asymptomatic. ${ }^{[4]}$ Therefore, a convenient, high-throughput and rapid test is urgently needed to widely screen a large number of patients with mild symptoms and asymptomatic carriers, for prompt quarantine and timely treatment.

Untill now, several antibody testing reagents have been approved by the National Medical Products Administration (NMPA) to assist the diagnosis of SARS-Cov-19 infection, including kits for testing total $\mathrm{Ab}$, IgM antibody or IgG antibody, by the methods of enzyme linked immunosorbent assay (ELISA), chemiluminescence or colloidal gold. In the diagnosis and treatment scheme for Corona Virus Disease 2019 (the seventh edition) from China which is just released on Mar 3, 2020, the detection of specific antibody was included as one of the etiological evidence to make the diagnosis. $^{[5]}$

The chemiluminescence method, ELISA, and colloidal gold method were used for detection of total $\mathrm{Ab}$ and IgM against SARS-CoV-2. The double-antigen sandwich method was applied for total $\mathrm{Ab}$ detection and $\mu$-capture method was applied for IgM detection (Table 1). The sensitivity and specificity for total Ab and IgM detection are high by all the three methods, and compared with $\operatorname{IgM}$, the sensitivity is higher for total $\mathrm{Ab}$ detection.

Table 1 . The sensitivity and specificity of total antibody and $\operatorname{IgM}$ antibody detected by different methods

\begin{tabular}{|c|c|c|c|}
\hline Method & Antibodies & Sensitivity & Specificity $^{2}$ \\
\hline \multirow{2}{*}{$\begin{array}{c}\text { Fully automatic tubular } \\
\text { chemiluminescence method }\end{array}$} & Total Ab & $86.9 \%(192 / 221)$ & $99.2 \%(358 / 361)$ \\
\cline { 2 - 4 } & IgM & $74.3 \%(165 / 222)$ & $99.2 \%(358 / 361)$ \\
\hline \multirow{2}{*}{ ELISA } & Total Ab & $94.8 \%(289 / 305)$ & $100 \%(333 / 333)$ \\
\cline { 2 - 4 } & $\operatorname{IgM}$ & $86.9 \%(265 / 305)$ & $100 \%(333 / 333)$ \\
\hline \multirow{2}{*}{ Colloidal gold method } & Total Ab & $96.2 \%(127 / 132)$ & $95.2 \%(199 / 209)$ \\
\cline { 2 - 4 } & $\operatorname{IgM}$ & $87.9 \%(116 / 132)$ & $98.1 \%(205 / 209)$ \\
\hline
\end{tabular}

Note. 1. The data in the brackets: the positive number/the number of tested patients; 2 . The data in the brackets: the negative number/the number of tested healthy persons.

In a study on SARS-CoV-2 antibody response in COVID-19 patients preprinted in March, 2020 by Shenzhen Third People's Hospital, Xiamen University, etc., 173 
confirmed patients were tested for total antibodies (Ab), IgM and IgG against SARS-CoV-2. The results showed that combined use of the tests of RNA and total Ab increased the diagnostic sensitivity to $99.4 \%$, comparing with that of $67.1 \%$ for the RNA only test. Even at the early stage of the disease course (within 7 days after looking for medical advice), the diagnostic sensitivity of the combination of RNA and total $\mathrm{Ab}$ was $78.7 \%$, which was $12 \%$ higher than the only test of RNA $(78.7 \%$ vs $66.7 \%$ ). Comparing with total $\mathrm{Ab}$, the sensitivity is lower for a single indicator of $\operatorname{IgM}$ or $\mathrm{IgG}$, or combining the 2 indicators of $\operatorname{IgM}$ and $\operatorname{IgG}$. Meanwhile, the specificity of the assays for total $\mathrm{Ab}, \operatorname{IgM}$ and $\mathrm{IgG}$ was determined as $99.1 \%, 98.6 \%$ and $99.0 \%$ by testing of samples collected from healthy individuals before the outbreak of SARS-CoV-2. Thus, the combined use of RNA and total Ab tests can improve the diagnostic sensitivity while remain the high specificity, which was thought to have great value for clinical practice and public health. ${ }^{[6]}$

According to a report in Science magazine on February 27, investigators in Singapore first applied $\mathrm{Ab}$ testing in tracking COVID-19 contact. ${ }^{[7]} \mathrm{After}$ the $\mathrm{Ab}$ were detected in an asymptomatic contact with negative nucleic acid test, the source of infection was successfully traced and the connection between two clustered cases was established. This breakthrough discovery also confirmed that serological testing was of great meaning for tracking the source with inapparent infections.

The diagnosis and treatment scheme for Corona Virus Disease 2019 (the seventh edition) from China indicates that the seropositivity of $\operatorname{IgM}$ and $\mathrm{IgG}$, seroconversion of $\mathrm{IgG}$, or 4-fold or greater increase in serum $\operatorname{IgG}$ titer in the recovery period compared with the acute period are the pathogenic evidence for confirming cases ${ }^{[5]}$. In general, IgM antibody is primarily induced and promptly reach a peak after infection, but the persistence time is relatively short. While the IgG antibody is mainly produced in the middle and late stages of the infection, and it can persistent for a long time at a high level even after recovery. Since SARS-CoV-2 is an emerging pathogen and has just infected human beings from Dec. 2019, which is less than 3 months from now, the prevalence of anti-SARS-CoV-2 antibodies in the general population is close to zero. In general, the double-antigen sandwich method used for total antibody detection has methodological advantages over $\mu$-capture (commonly used for $\operatorname{IgM}$ antibody detection) and indirect ELISA method (commonly used for IgG antibody detection): 1) Simultaneous detect IgM, IgG, IgA and other types of antibodies; 2) Detection request the two Fab fragments of the antibody molecule respectively bound to the coating antigen and labeled antigen, and this characteristic significantly reducing the nonspecific binding caused by the heterogeneous proteins in the coating antigen and the labeled antigen, thus ensuring higher specificity. 3) Due to the very limited interference of heterologous proteins in the antigen, increasing the concentration of the coating antigen and labeled antigen can further improve the sensitivity of the detection. Zhao et al. performed antibody testing on 535 series of plasma from 173 confirmed patients at different time points after the onset of COVID-19. ${ }^{[6]}$ Based on the methodological advantages, the average seroconversion 
time of total $\mathrm{Ab}$ was 1 day and 3 days earlier than $\operatorname{IgM}$ and $\operatorname{IgG}$ respectively, and the signal-to-noise ratio of the positive response was also higher. The above suggests that the total Ab could increase diagnostic sensitivity meanwhile keep the specificity, and may be a better serological diagnostic indicator for early diagnosis. The study from Li et al. also reported that the sensitivity of combining IgM with IgG was higher than applying $\operatorname{IgM}$ or IgG alone, while the specificity remain similar. ${ }^{[8]}$

Overall, testing $\mathrm{Ab}$ by methods of ELISA and chemiluminescence has the characteristics of convenient sampling, high-throughput, high efficiency and can be quantitative or semi-quantitative, and method of colloidal gold is a real-time and point-of-care testing. The above methods of Ab testing should be selected as an effective supplement for RNA detection under different circumstances, to improve the diagnostic sensitivity and specificity. The applications of Ab testing can be summarized as following:

Screening and Management of Clinical Patients and Close Contacts: 1) for the suspected patients and clinically diagnosed patients who have negative RNA testings, COVID-19 diagnosis can be made with positive $A b$; 2) with positive $A b$, the asymptomatic close contact should be deemed as a current carrier or being previous infected. For close contact, if RNA testing of the upper respiratory tract specimen remains to be negative during the 14 days of quarantine, positive Ab implies more days of quarantined observation and further collection and nucleic acid testing for lower respiratory tract and stool specimens should be considered; 3) Ab testing can be used to quantitatively measure antibody levels in plasma of recovery patients, considering that plasma with high-titer antibody may be helpful for treatment of severe COVID-19 patient.

Screening of Key Populations: A combination of RNA and Ab testing can be applied to screen for travelers from countries or regions with high incidence of COVID-19, participants of important conferences or events, and population returning back work or school from high incidence areas. For people with negative RNA while positive $A b$, quarantine and health monitoring may also be conducted to timely identify potential cases and decrease the risk of transmission.

Epidemiology Research: 1) by combining the serological test with nucleic acid test and clinical symptom, to analyze the full spectrum of SARS-CoV-2 infection, revealing the distribution of inapparent infection, atypical infection, mild symptom infection, severe symptom infection, and the contribution of each kind of the infection to the transmission of virus and disease; 2) conduct population-based serological survey, to understand the actual prevalence and pathogenicity of SARS-CoV-2 infection in different regions and populations, such as population of different ages, occupations or with different underlying disease, thus, suggest appropriate disease control interventions ; 3 ) collect series of samples from suspected cases or confirmed 
cases and test for serological indicators, nucleic acid indicators and other laboratory indicators, to analyze their dynamics, thus, improve COVID-19 diagnosis and treatment strategy; 4) analyze the antibody level and spectrum of antibody epitopes in convalescent COVID-19 patients, to provide scientific guidence for the design and evaluation of vaccines and therapeutic antibodies in the future; 5) support the search for potential animal hosts of SARS-CoV-2, as the total Ab detected by the double-antigen sandwich method is not restricted to species.

\section{Competing Interest Statement}

The authors have declared no competing interest.

\section{Funding}

This study is supported by a grant from the Bill \& Melinda Gates Foundation. 


\section{References}

[1] World Health Organization. Coronavirus disease 2019 (COVID-19) situation report-47 [EB/OL].(2020-03-07)[2020/03/10].

https://www.who.int/docs/default-source/coronaviruse/situation-reports/20200305-sitrep-45-covid-19.p df? sfvrsn=ed2ba78b 2.

[2] World Health Organization. Coronavirus disease 2019 (COVID-19) situation report-39 [EB/OL]. (2020-02-28)[2020/03/10].

https:/www.who.int/docs/default-source/coronaviruse/situation-reports/20200228-sitrep-39-covid-19.p df? sfvrsn=5bbf3e7d 2.

[3] Guan W-J, Ni Z-Y, Hu Y, et al. Clinical Characteristics of Coronavirus Disease 2019 in China [J]. New England Journal of Medicine, 2020. DOI:10.1056/NEJMoa2002032.

[4] $\mathrm{Wu} \mathrm{Z,} \mathrm{Mcgoogan} \mathrm{J} \mathrm{M.} \mathrm{Characteristics} \mathrm{of} \mathrm{and} \mathrm{Important} \mathrm{Lessons} \mathrm{From} \mathrm{the} \mathrm{Coronavirus} \mathrm{Disease}$ 2019 (COVID-19) Outbreak in China: Summary of a Report of 72314 Cases From the Chinese Center for Disease Control and Prevention [J]. JAMA, 2020. DOI:10.1001/jama.2020.2648.

[5] National Health Commission of China. The diagnosis and treatment scheme for Corona Virus Disease 2019 (the seventh edition) [EB/OL].2020[2020-03-10]. http://www.nhc.gov.cn/yzygj/s7653p/202003/46c9294a7dfe4cef80dc7f5912eb1989/files/ce3e6945832a 438eaae415350a8ce964.pdf.

[6] Zhao J, Yuan Q, Wang H, et al. Antibody responses to SARS-CoV-2 in patients of novel coronavirus disease 2019 [J]. medRxiv, 2020:2020-2023. DOI:10.1101/2020.03.02.20030189.

[7] Normile D. Singapore claims first use of antibody test to track coronavirus infections [N]. Science, (2020-02-27)[2020-03-08].

https://www.sciencemag.org/news/2020/02/singapore-claims-first-use-antibody-test-track-coronavirus-i nfections.

[8] Hui L, Yongyin L, Zhigao Z, et al. Establishment and clinical performance evaluation of 2019 novel coronavirus antibody colloidal gold detection method [J]. Chinese Journal of Infectious Diseases, 2020(00):E17 